\title{
Visualization of the variance boundaries for the polarization parameters of light on the Poincare sphere
}

\author{
V.I. Smirnov \\ National Research University "MPEI" \\ ORCID: 0000-0002-3047-988X, smirnovvi-mpei@yandex.ru
}

\begin{abstract}
$\underline{\text { Abstract }}$
The methods of parametric information theory are used to study the minimal variance boundaries of polarization parameters estimates of partially polarized quasi-monochromatic radiation in a polarimetric scheme with an analyzer and a phase compensator under conditions of additive-multiplicative normal noise. The density distributions of information on the Stokes parameters in the coordinates of the angle of rotation of the analyzer and the phase shift of the compensator are shown. The analysis of these distributions allows finding the most favorable measurement plans in metrological relation. Matrix variance boundaries of different polarization parameters for the uniform plan and other measurement plans are calculated. The error limits of the estimates of the degree of polarization are calculated. The results of the analysis are confirmed by numerical simulation. The patterns of the distributions of the variance bound estimates of the degree of polarization on the Poincare sphere are given. With the help of the developed technique based on the parametric information theory, it is possible to study estimates of any polarization parameters: azimuth, ellipticity and others. The results of this study can be used to optimize signal processing algorithms and improve the efficiency of optoelectronic devices and systems of a wide profile.
\end{abstract}

Keywords: polarized measurements, effective estimation, Stokes parameters, degree of polarization, variance boundary visualization.

\section{Introduction}

The use of polarization devices in laser technology, in optical devices and systems makes the problem of precision measurement of the polarization characteristics of radiation relevant. There are different ways of measuring the polarization state [1]. The set of devices realizing them is invented. In [2-3] metrological features of polarization measurements planning in conditions of additive noise are considered. In this paper we generalize the results to the case of additive-multiplicative normal noise. The polarization state of a quasi-monochromatic wave can be specified in different ways. In many cases, it is convenient to use Stokes parameters that have the same dimension, real values, and have a visual interpretation of the coordinates of the point on the Poincare sphere [1]. Stokes parameters are also used in crystallography and quantum physics.

\section{Basic relations}

Analysis of polarization measurements can be performed using a registration scheme with a polarizer and a phase compensator, in which it is possible to scan the angle of rotation of the polarizer $\theta$ and the phase delay $\varepsilon$ introduced by the compensator. The input receives the analyzed radiation, the intensity of the transmitted wave is measured on the output. The parametric model of the problem is the functional dependence of the output value on the factors $\theta, \varepsilon$ and the required Stokes parameters of the input radiation. This model is given in [2] and has the form 


$$
I(\theta, \varepsilon ; \mathbf{s})=\frac{s_{0}}{2}+\frac{s_{1}}{2} \cos (2 \theta)+\frac{s_{2}}{2} \sin (2 \theta) \cos (\varepsilon)+\frac{s_{3}}{2} \sin (2 \theta) \sin (\varepsilon) .
$$

Formula (1) defines a 2-factor 4-parametric model. Factors: $\theta$ - the rotation angle of the analyzer, $\varepsilon-$ the phase shift specified by the compensator. The parameters of the model are the Stokes parameters indicated by the vector $\mathbf{S}=\left(s_{0}, s_{1}, s_{2}, s_{3}\right)$.

The visual interpretation of the Stokes parameters of fully polarized radiation is related to the use of the Poincare sphere [1]. For partially polarized radiation, the Poincare sphere can be conventionally represented as shown in Fig.1.

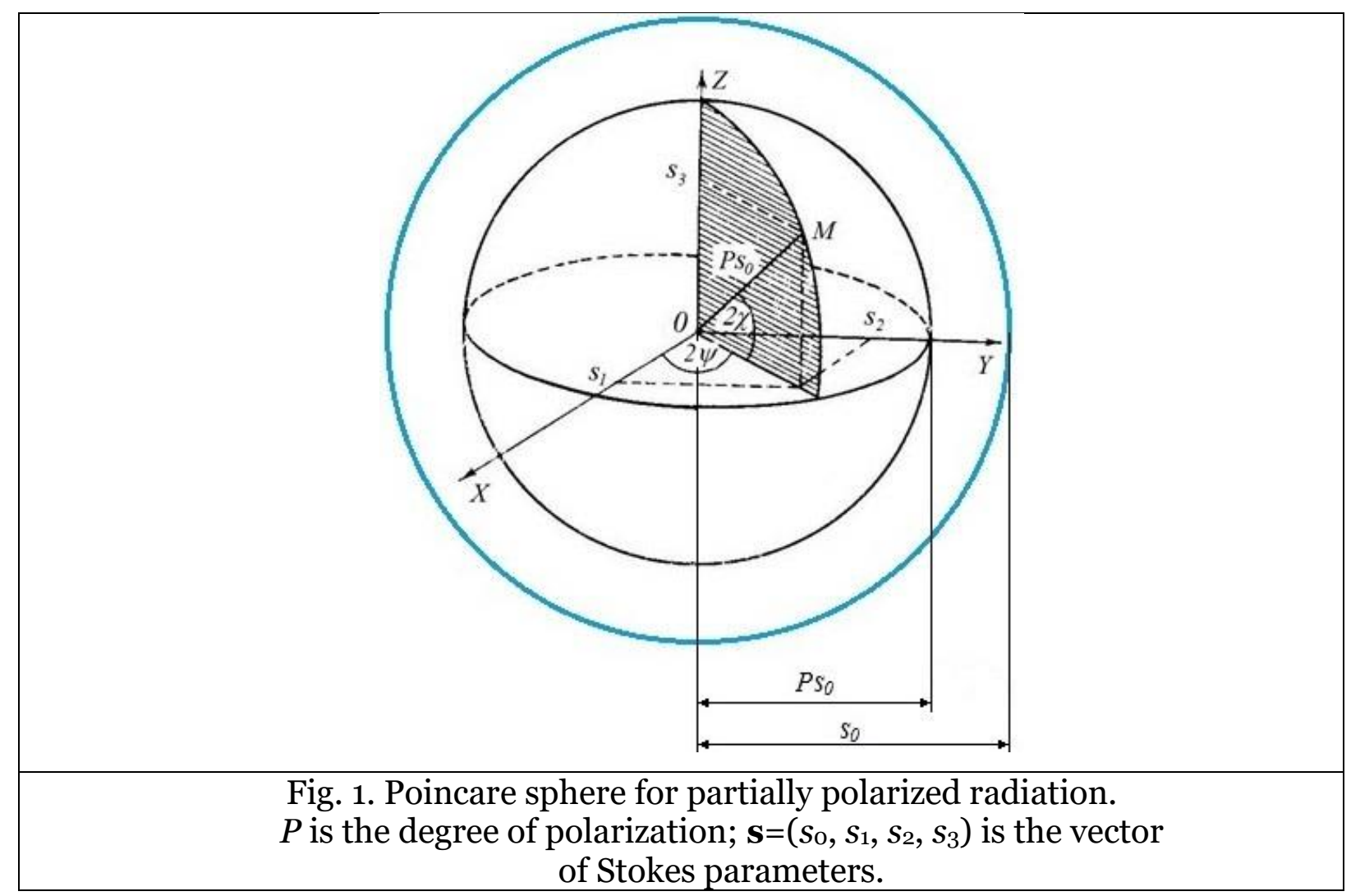

The position of the point $\mathrm{M}$ characterizes the polarization state of the polarized component. Angle $2 \psi$ - longitude and $2 \chi$ - latitude of the point M. From the point of view of the shape and orientation of the polarization ellipse angle $\psi$ is the angle of inclination of the major axis of the ellipse to the $\mathrm{x}$-axis, and $\tan (\chi)$ is equal to the ratio of the minor and major axis. Thus, the States with linear polarization are located at the equator, and the States with right and left circular polarization are located at the North and South poles of the Poincare sphere. From Fig.1 you can see that

$$
s_{1}=s_{0} P \cos (2 \chi) \cos (2 \psi), \quad s_{2}=s_{0} P \cos (2 \chi) \sin (2 \psi), \quad s_{3}=s_{0} P \sin (2 \chi) .
$$

According to the Rao-Kramer theorem [4], there is a lower boundary for the variance matrix of model parameter estimates. It determines the theoretical limit accuracy of the joint estimation of parameters, potentially achievable with a given statistics of measurement errors if one uses all information obtained from the experiment. Variance matrix boundary can be found as the inverse of the information matrix . As follows from the formulas given in [2], when measured at arbitrary points with coordinates $\left(\theta_{k}, \varepsilon l,\right)(k=0,1, \ldots m ; l=0,1, \ldots n)$ elements of the information matrix can be represented as

$$
I M_{i j}=\sum_{k=0}^{m-1} \sum_{l=0}^{n-1} \frac{1}{D_{k, l}} \frac{\partial}{\partial s_{i}}\left[I\left(\theta_{k}, \varepsilon_{l} ; \mathbf{s}\right)\right] \frac{\partial}{\partial s_{j}}\left[I\left(\theta_{k}, \varepsilon_{l} ; \mathbf{s}\right)\right],
$$


where $D_{k, l}$ characterizes the noise power. For additive-multiplicative noise with $D_{k, l}$ normalization selected in [2], can be represented as

$$
D_{k, l}=D\left(\mu, \theta_{k}, \varepsilon_{l} ; \mathbf{s}\right)=1-\mu+\mu \frac{I\left(\theta_{k}, \varepsilon_{l} ; \mathbf{s}\right)}{s 0},
$$

where $\mu$ is the degree of multiplicativity of noise or the ratio of the power of the multiplicative component of noise to the total power of noise.

Formulas $(3,4)$ allow to calculate the information matrix for any set of points in the plane $(\theta, \varepsilon)$. Next, we evaluate the normalized matrices fitted to the unit total time of measurement $\mathrm{T}$ and the unit intensity $(\mathrm{sO}=1)$ of the analyzed radiation at the input of the measuring scheme. With a uniform distribution of measurement points on $\theta$ and $\varepsilon$ such that $\theta_{k}=\pi k / m, k=0, \ldots, m-1 ; \quad \varepsilon_{l}=\pi l / n, l=0, \ldots, n-1, m \geq 3, n \geq 2$, the elements of the normalized information matrix of the estimates of the Stokes parameters IN can be represented in the form

$$
I N_{i, j}=\frac{1}{m \cdot n} \sum_{k=0}^{m-1} \sum_{l=0}^{n-1} \frac{1}{1-\mu+\mu I\left(\theta_{k}, \varepsilon_{l} ; \mathbf{s}\right)} \frac{\partial}{\partial s_{i}}\left[I\left(\theta_{k}, \varepsilon_{l} ; \mathbf{s}\right)\right] \frac{\partial}{\partial s_{j}}\left[I\left(\theta_{k}, \varepsilon_{l} ; \mathbf{s}\right)\right]
$$

At the unlimited magnification of $n$ and $m$ we come to the integral expression

$$
I N_{i, j}=\int_{0}^{\pi} \int_{0}^{\pi} \frac{1}{\pi^{2}} \frac{1}{1-\mu+\mu I(\theta, \varepsilon ; \mathbf{s})} \frac{\partial}{\partial s_{i}}[I(\theta, \varepsilon ; \mathbf{s})] \frac{\partial}{\partial s_{j}}[I(\theta, \varepsilon ; \mathbf{s})] d \theta d \varepsilon
$$

\section{Information density}

The integrand function in (6) can be considered as the density of information for Stokes parameters in the plane $(\theta, \varepsilon)$ :

$$
w s_{i, j}(\theta, \varepsilon, \mathbf{s})=\frac{1}{\pi^{2}} \frac{1}{1-\mu+\mu I(\theta, \varepsilon ; \mathbf{s})} \frac{\partial}{\partial s_{i}}[I(\theta, \varepsilon ; \mathbf{s})] \frac{\partial}{\partial s_{j}}[I(\theta, \varepsilon ; \mathbf{s})]
$$

Examples of visualization of density distributions of information in the form of maps with a topographic color scale are shown in figure 2. Information density values $w s$ are normalized by the sum of the minimum and maximum ws values for this map, the intensity at the input of the measuring system is reduced to unit ( $\mathrm{sO}=1)$. The color scale is shown in figure 3 .

Figure 4 shows a series of information density maps for the Stokes parameters for a range of values of noise multiplicativity $\mu$. Format of axis corresponds to the format of the axis in figure 2.

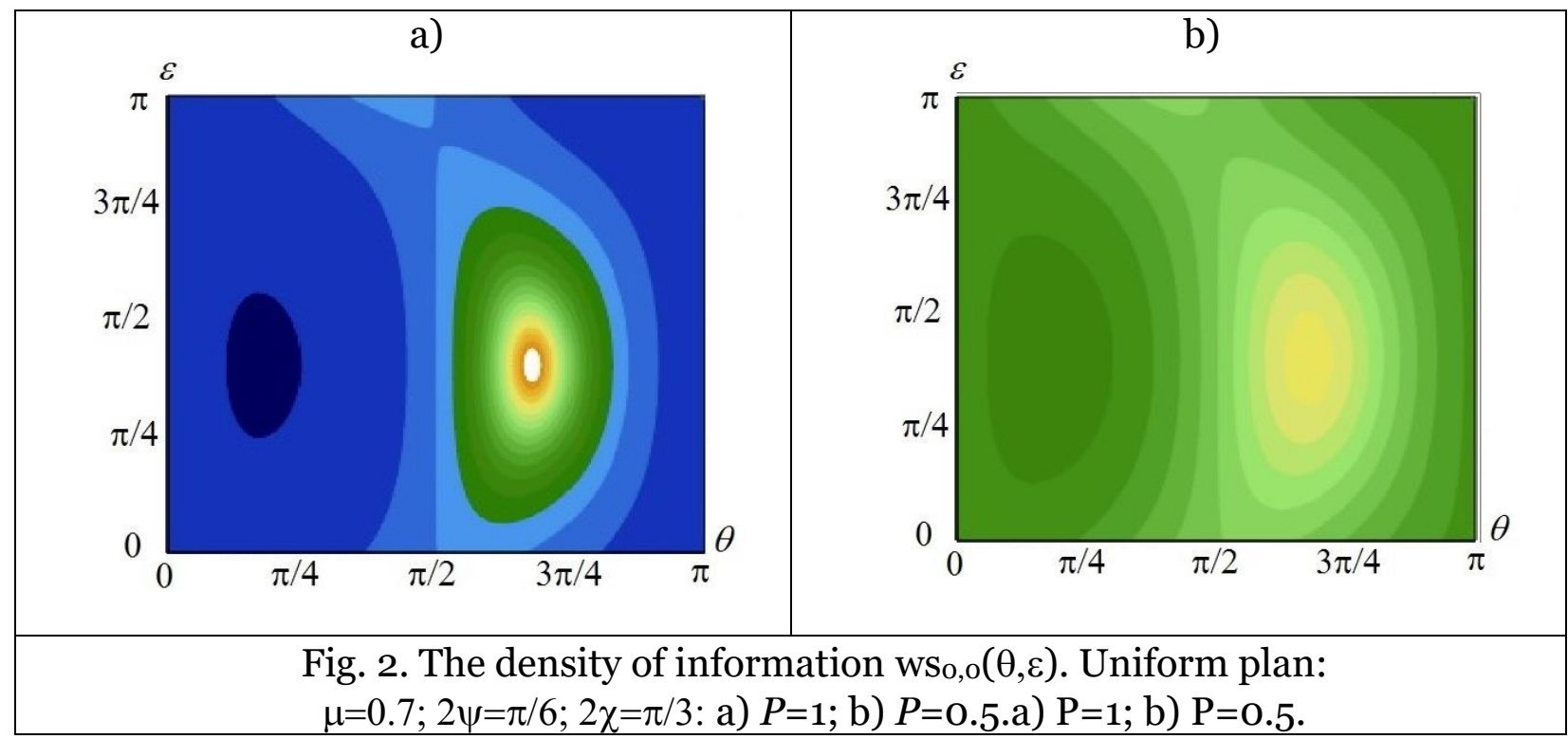



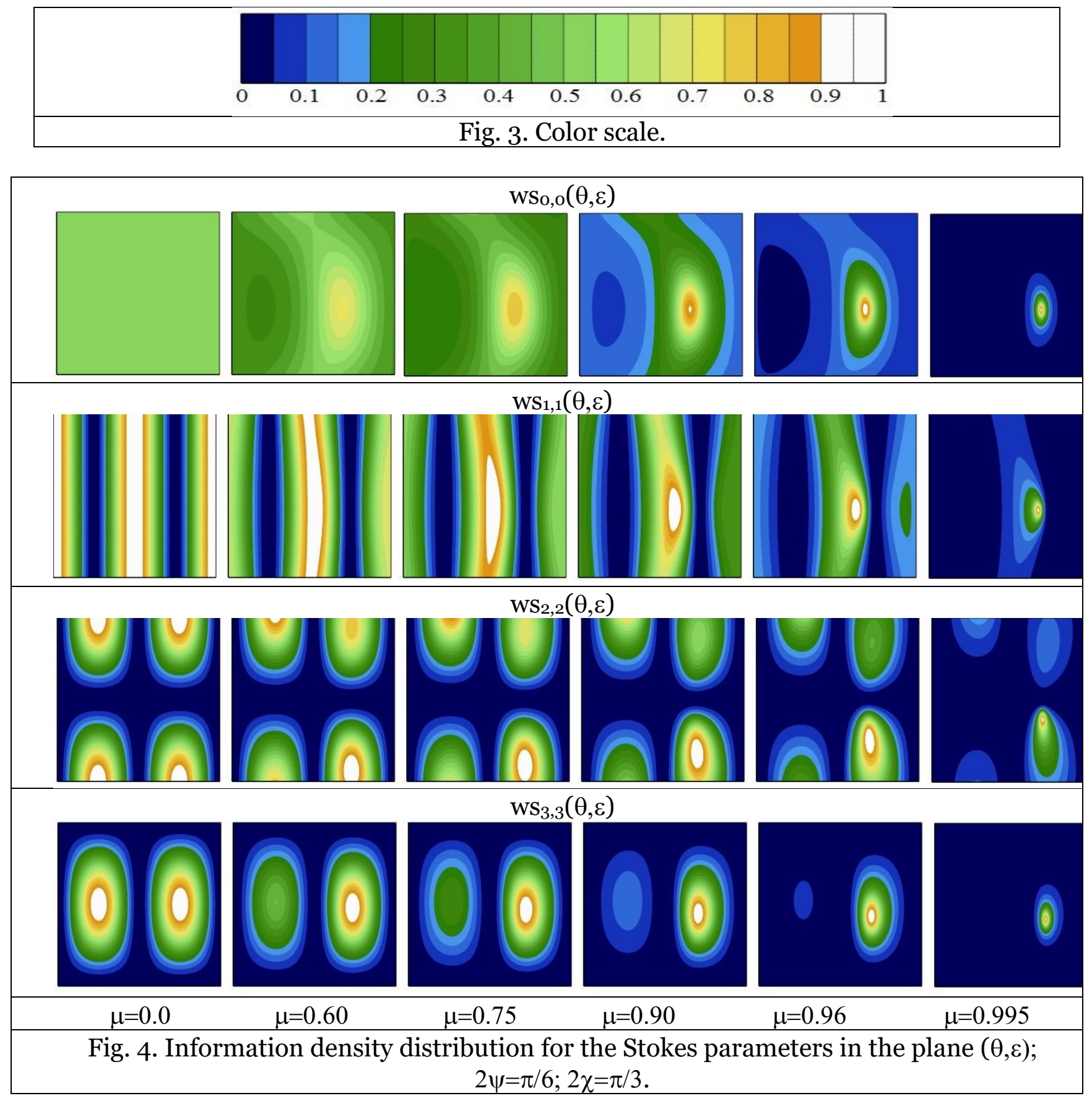

Analysis of distributions (Fig.4) allows one to find the best measuring plans in the metrological respect, that is to determine the coordinates and the number of measurement points in the plane $(\theta, \varepsilon)$ that will ensure maximum accuracy of the estimates of the Stokes parameters for the given parameters of the noise. Thus, for example, in [3], a justification was given for the 6-point optimal plan (presented in table 1 below) for the case of the additive normal noise.

\section{Visualization of vairiance boundaries}

The normalized dispersion matrix boundary of the Stokes parameter estimates is defined as the inverse of the normalized information matrix IN (5):

$$
\mathbf{D N}=(\mathbf{I N})^{-1}
$$

Any polarization parameter can be expressed as a function of Stokes parameters

$$
p=p\left(s_{0}, s_{1}, s_{2}, s_{3}\right)=p(\mathbf{s})
$$



follows

For the normalized minimum variance of the parameter $p$ estimate from (8) and (9) it

$$
D N p(\mathbf{s})=\sum_{k=0}^{3} \sum_{l=0}^{3}\left(\frac{\partial p(\mathbf{s})}{\partial s_{k}}\right)\left(\frac{\partial p(\mathbf{s})}{\partial s_{l}}\right) D N_{k, l}
$$

In this paper, we analyze the variance boundaries of the degree of polarization estimate $P$, a parameter of partially polarized radiation, which is expressed in terms of Stokes parameters as follows:

$$
P=\frac{\sqrt{s_{1}^{2}+s_{2}^{2}+s_{3}^{2}}}{s_{0}}
$$

Taking into account (2) and using the ratio (5, 8-11) we can get a visual picture of the variance bounderies of the estimate distributions of the degree of polarization on the Poincare sphere in Cartesian rectangular coordinates, where the abscissa corresponds to the longitude $2 \psi$, and the ordinate to the latitude $2 \chi$. With this representation, the spherical surface is transformed into a rectangle on the plane, the meridians are transformed into parallel vertical lines, and the parallels are stretched the more, the closer they are to the poles. Pole points turn into horizontal lines. Fig.5a and Fig.5b show an example of such a visualization of the variance boundareies of estimates of the polarization degree $\operatorname{DNP}(2 \psi, 2 \chi)$ using formulas $(2,5), 6$-point uniform plan $(\mathrm{m}=3, \mathrm{n}=2)$ and the same degree of the noise multiplicativity. The difference is only in the degree of polarization: a) $\mathrm{P}=1$; b) $\mathrm{P}=0.5$.

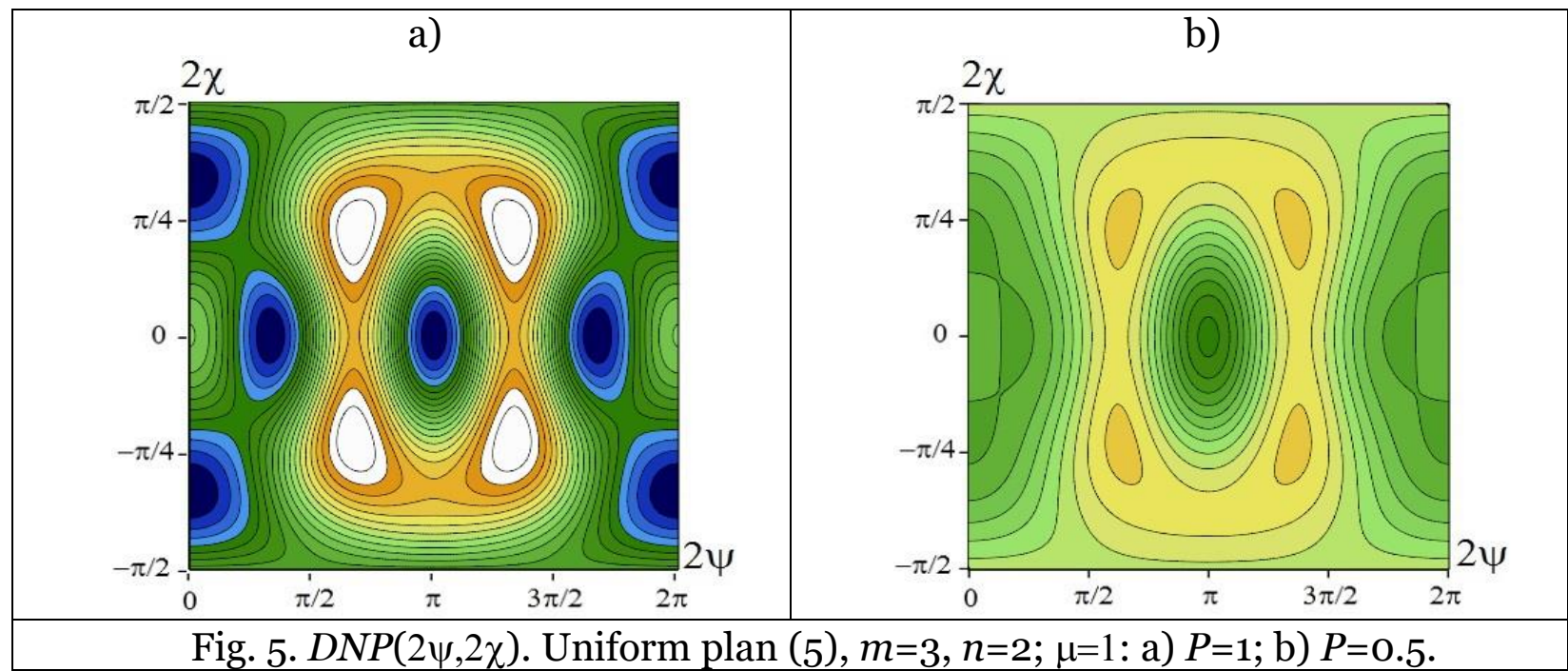

From the maps of variance boundaries shown in Fig. 6, one can see the nature of the change $\operatorname{DNP}(2 \psi, 2 \chi)$ for different combinations of $m$ and $n$ at a fixed degree of noise multiplicativity $\mu=1$. 


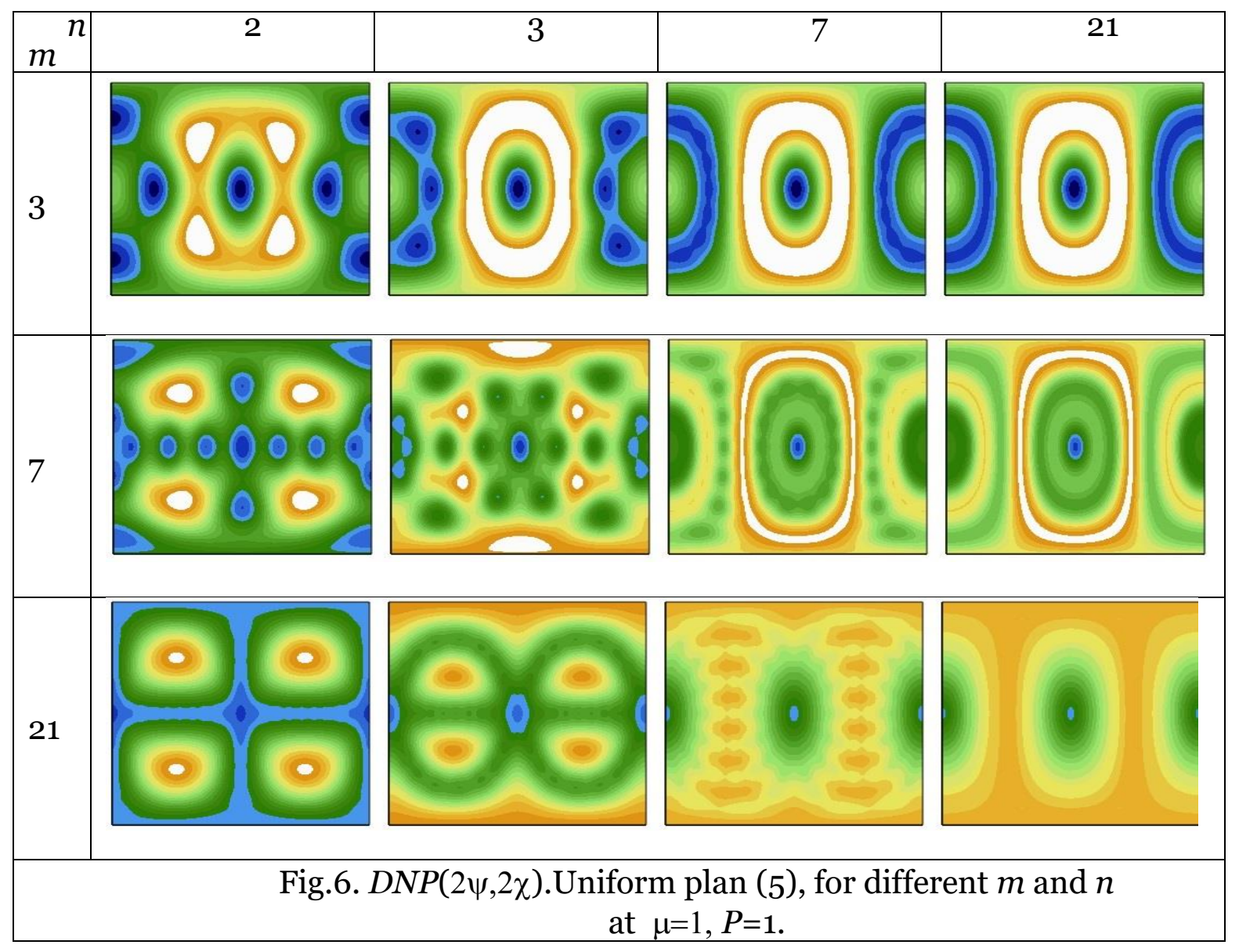

Animation 1 illustrates in more detail the changes in the variance boundaries with an increase in the number of measurement points at $n=m$ and $\mu=1$.

In [2-3] based on the analysis of density distributions of information (see Fig.4) it is shown that at additive noise $(\mu=0) 6$-point plan is the optimum. It is optimal in respect to the criterion of minimum of the normalized dispersion, and can be presented in the form of table 1. Such a plan is heuristically proposed in the monograph [1] without the corresponding error analysis.

Table 1. The optimal plan $6 \mathrm{pO}$

\begin{tabular}{|l|l|l|l|l|l|l|}
\hline № & $\mathrm{O}$ & $\mathbf{1}$ & $\mathbf{2}$ & 3 & 4 & 5 \\
\hline$\theta$ & $\mathrm{O}$ & $\pi / 2$ & $\pi / 4$ & $3 \pi / 4$ & $\pi / 4$ & $3 \pi / 4$ \\
\hline$\varepsilon$ & $\mathrm{O}$ & $\mathrm{O}$ & $\mathrm{O}$ & $\mathrm{O}$ & $\pi / 2$ & $\pi / 2$ \\
\hline
\end{tabular}

Let us consider the distribution of the variance boundary estimates of polarization degree $P$ on the Poincare sphere for 6-point optimum plan for $6 \mathrm{pO}$ (Fig.1), as well as for its $5^{-}$ point $5 \mathrm{pO}$ and 4-point $4 \mathrm{pO}$ shortened variants, under additive-multiplicative normal noise conditions. Imaging results of the visualization of $D N P(2 \psi, 2 \chi)$ are shown in figures 7-9 with the use of topographic scale (Fig.2) with the same normalization.

Fig.7 shows a series of maps $\operatorname{DNP}(2 \psi, 2 \chi)$ for the plan $6 \mathrm{pO}$ at different $\mu$. For comparison, similar series of distributions for a uniform plan (5) with $\mathrm{m}=3, \mathrm{n}=2$ are shown in the same table in the second line under the code $6 \mathrm{pU}$. 


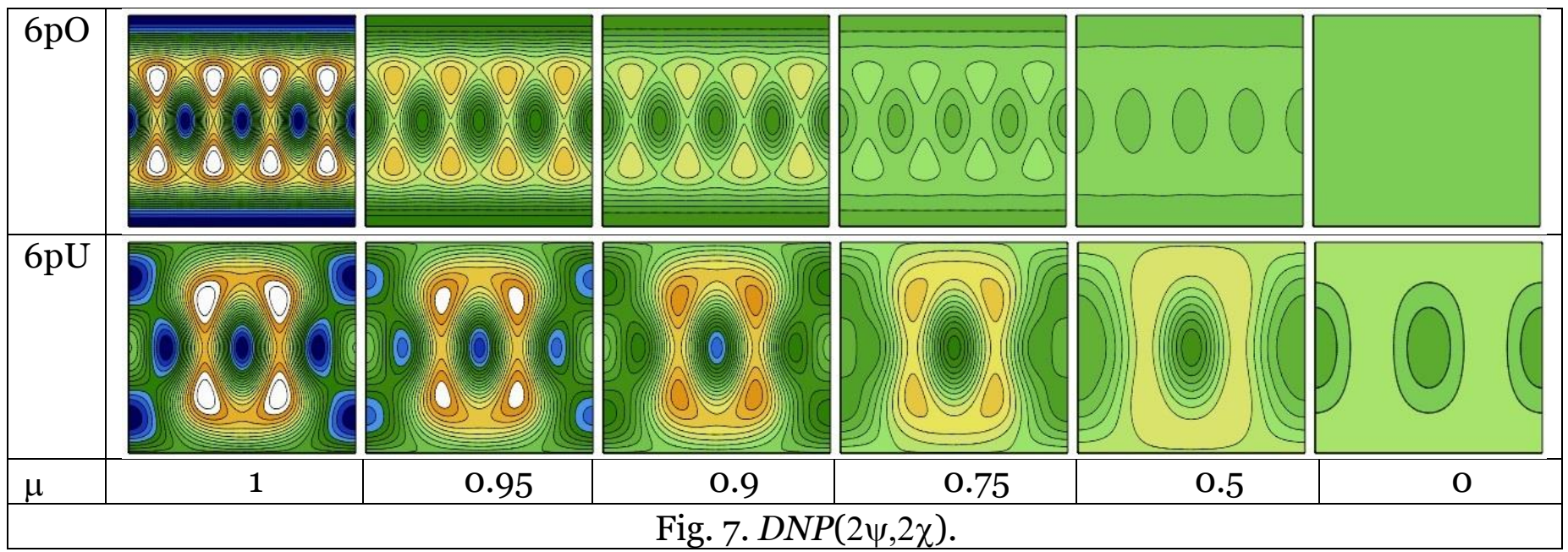

In more detail the variance boundary change is illustrated in the dynamics by animations 2 and 3 while $\mu$ changes from 1 to 0 .

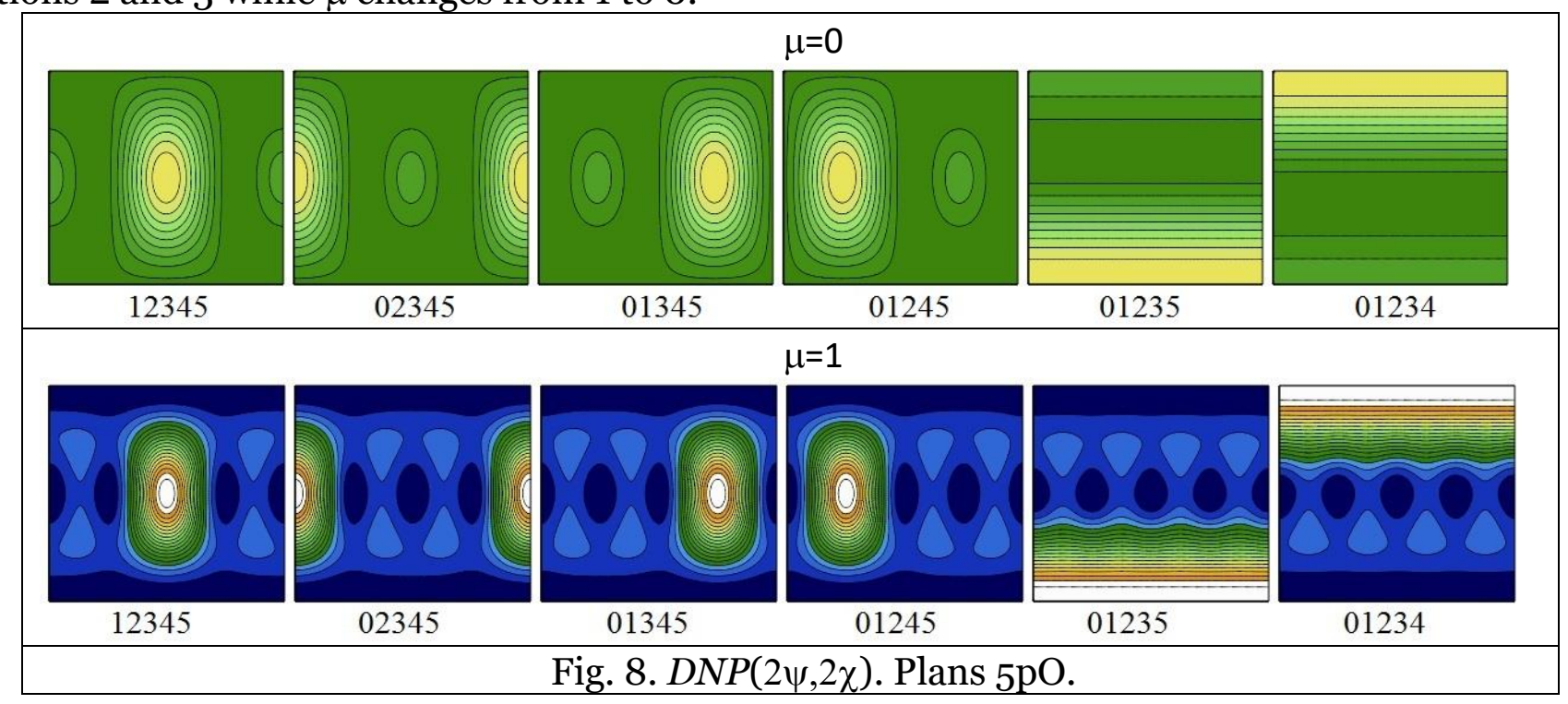

Fig. 8 presents the pictures of the distributions $\operatorname{DNP}(2 \psi, 2 \chi)$ with $\mu=0$ and $\mu=1$ for all of 6 possible 5-point plans obtained by removing one point from the table. 1 . The five-digit code here corresponds to the measurement point numbers.

Using the presented figures one can understand the influence of various points of the optimal plan on the nature of the transformation of the patterns. Thus, replacing 4 to 5 leads to the conversion of paintings relative to the equator of the Poincare sphere; 2 to 3 - to conversion relative to the Central Meridian; o to 1 - to shift along the equator in $\pi$.

Fig. 9 shows the pattern for $\operatorname{DNP}(2 \psi, 2 \chi)$ with $\mu=0$ and $\mu=1$ for all 12 possible 4-point plans obtained by removing two points of the table. 1 . The four-digit code here corresponds to the measurement point numbers. 


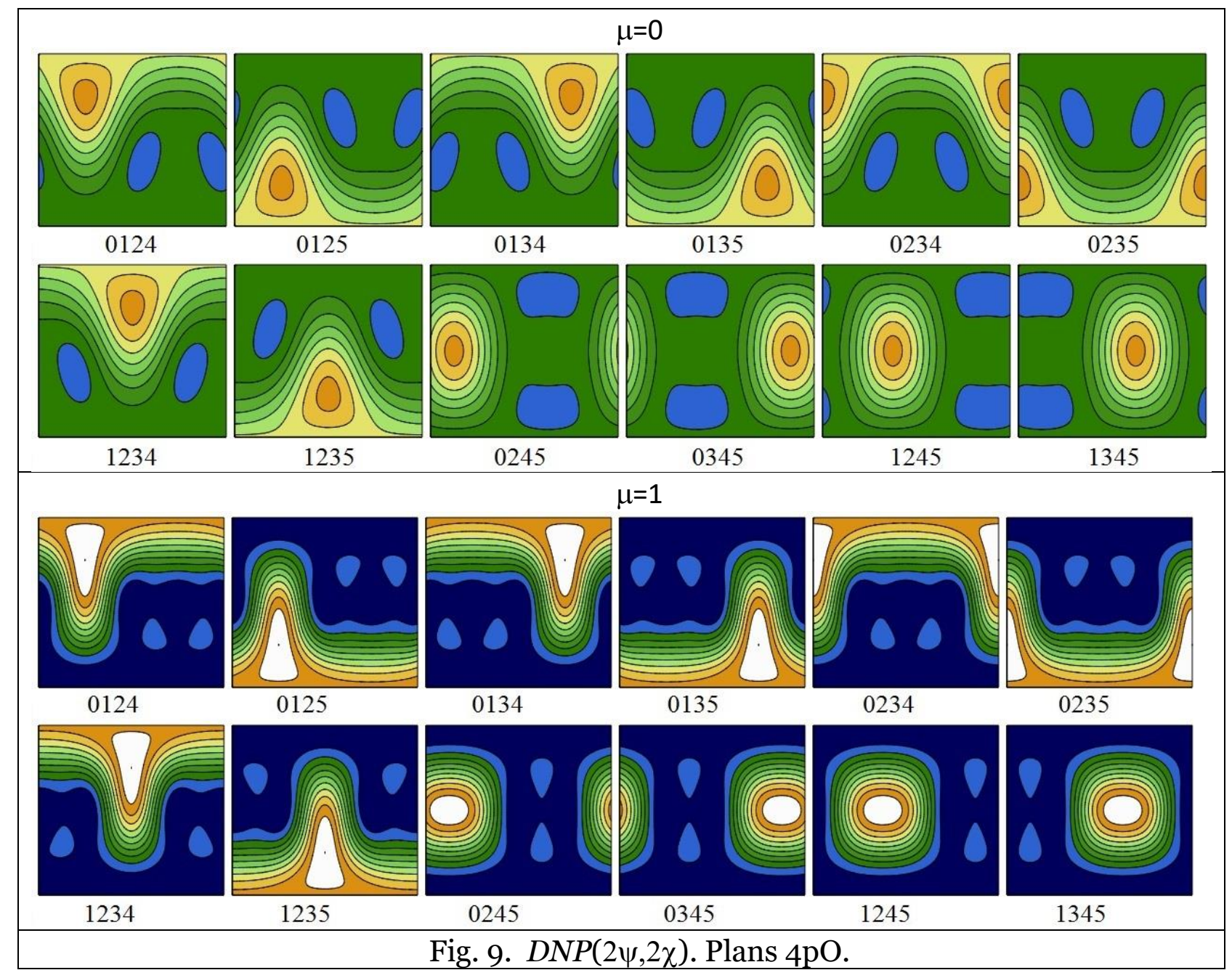

In practice, along with the optimal 6-point plan, 4-point plans and a plan that can be expressed by the formula $3 \mathrm{pO}+\mathrm{so}$ [5] are often used. The latter consists of three measurements at the coordinates from the table 1 and one measurement of the total radiation intensity so.

Fig. 10 shows patterns for $\operatorname{DNP}(2 \psi, 2 \chi)$ when $\mu=0$ and $\mu=1$ for all 8 possible 4-point plans $3 \mathrm{pO}+\mathrm{so}$. The three-digit code here corresponds to the measurement point numbers from the table. 1.

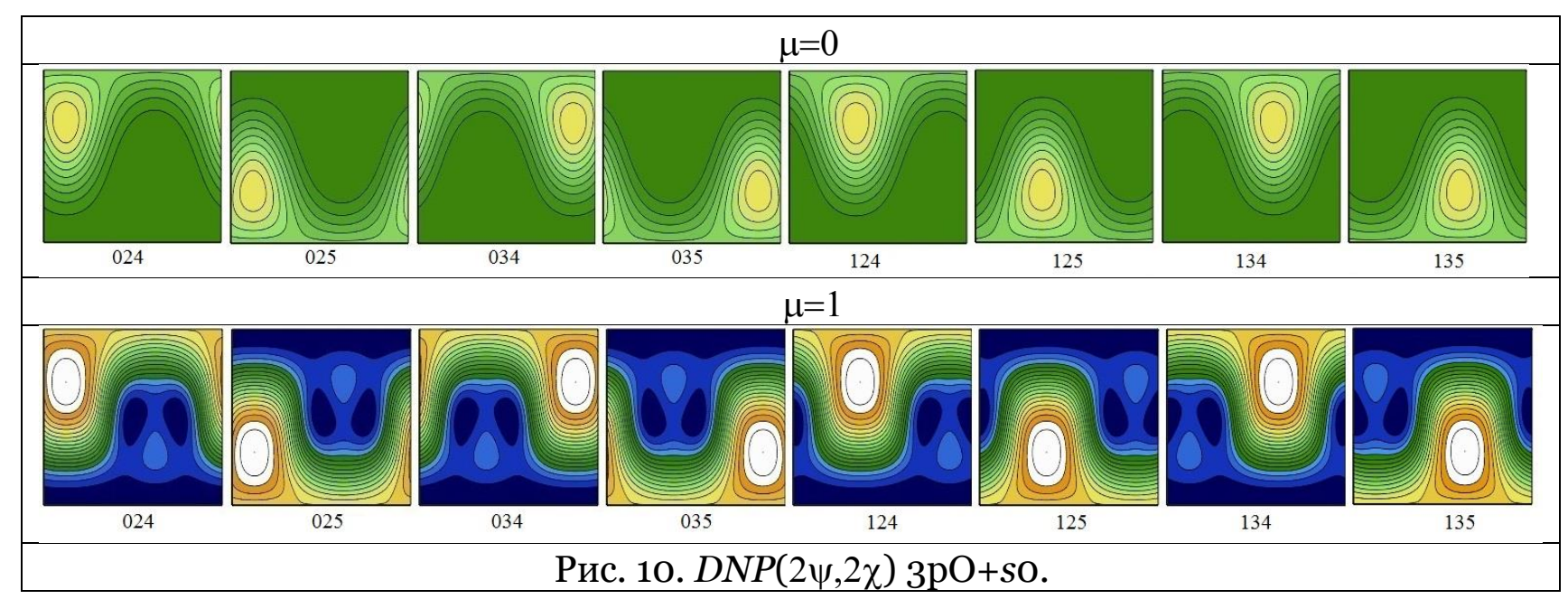


The figures show that the areas with potentially minimal measurement errors change their position on the Poincare sphere depending on the code. In the presence of a priori information it allows to optimize the choice of points for measurements.

\section{The ranges of values of $|D N|$ and $D N P$}

Ranges of $|\mathrm{DN}|$ and DNP values for the above plans are presented in table 2 at $P=1$ and different values $\mu$. The $6 \mathrm{pU}$ cipher is used to denote a 6-point uniform plan (5) at $m=3$, $n=2$ (see Fig. 5a). Table 3 gives the example of the plan 4 pO showing how the boundaries of the ranges for $|\mathrm{DN}|$ and $D N P$ depend on the degree of polarization $P$.

\begin{tabular}{|c|c|c|c|c|c|c|}
\hline & \multicolumn{3}{|c|}{$|\mathbf{D N}|$} & \multicolumn{3}{|c|}{$D N P$} \\
\hline$\mu$ & $\mathrm{O}$ & 0.75 & 1 & $\mathrm{O}$ & 0.75 & 1 \\
\hline $6 \mathrm{pU}$ & $8.192 \cdot 10^{3}$ & $675-1167$ & $0-336$ & $12-20$ & $3.0-10.7$ & $0-7.9$ \\
\hline $6 \mathrm{pO}$ & $6.912 \cdot 10^{3}$ & $675-719$ & $0-128$ & 16 & $5.2-7.0$ & $0-4.0$ \\
\hline $5 \mathrm{pO}$ & $1.000 \cdot 10^{4}$ & $610-2444$ & $0-1.3 \cdot 10^{3}$ & $13-40$ & $4.4-32.5$ & $0-30$ \\
\hline $4 \mathrm{pO}$ & $1.638 \cdot 10^{4}$ & $829-5071$ & $0-3.0 \cdot 10^{3}$ & $11-63$ & $3.6-43.4$ & $0-37$ \\
\hline $3 \mathrm{pO}+\mathrm{so}$ & $1.638 \cdot 10^{4}$ & $1117-9761$ & $0-8.0 \cdot 10^{3}$ & $16-46$ & $4.0-43.0$ & $0-42$ \\
\hline
\end{tabular}

\begin{tabular}{|c|c|c|c|c|c|c|}
\hline & & $|\mathbf{D N}|$ & & & $D N H$ & \\
\hline $\begin{aligned} & P \\
\mu & \end{aligned}$ & O & 0.75 & 1 & O & 0.75 & 1 \\
\hline 0 & $1.638 \cdot 10^{4}$ & $1.638 \cdot 10^{4}$ & $1.638 \cdot 10^{4}$ & $11-63$ & $8.7-45$ & $8-32$ \\
\hline 0.75 & $829-5071$ & $1525-3675$ & $1066-1066$ & $3.6-43.4$ & $4.6-31$ & $5-20$ \\
\hline 1 & $0-2984$ & $428-1876$ & $1924-1024$ & $0-37$ & $3-25$ & $4-16$ \\
\hline
\end{tabular}

The obtained results were verified by the method of numerical statistical experiment, in which the noise was modeled by a random number generator with normal additivemultiplicative noise [6].

\section{Conclusion}

In conclusion, we note that with the help of the developed technique based on the parametric information theory, it is possible to study the estimates of any polarization parameters: azimuth, ellipticity, and others. The results of this study can be used to optimize signal processing algorithms and improve the efficiency of optoelectronic devices and systems of a wide profile: from laser Doppler flow velocity meters [7] and quantum optical guidance systems $[8,9]$ to astronomical $3 \mathrm{D}$ interferometry systems $[10,11]$.

\section{References}

1. Born M., Wolf E. Principles of optics. M: Nauka, 1973.

2. Smirnov V.I. Effective Estimates of the Stokes Parameters of Partially Polarized Radiation in Conditions of Normal Additive Noise. Measurement Techniques: January 2019, Volume 61, Issue 10, Page 1001-1009.

3. Smirnov V. I. Analysis of errors in measuring the polarization parameters of quasimonochromatic radiation on the basis of information theory $\backslash \backslash$ Optical methods for studying flows: XIV Inter. science and technology. conference [Electronic resource]: proceedings of the conference. - Electron. dat. - M.: national research UNIVERSITY "MPEI", 2017. Pp. 346-363. ISBN 978-5-906972-44-6. 
4. Bard Y. Nonlinear parameter estimation. M: Statistica, 1974.

5. IshchenkoE. F., Sokolov A. L., Polarization analysis. M.: Publishing House "Znak". 1998.

6. Smirnov V. I., Yanina G. M. Analysis of potential accuracy of measurement of polarization parameters of partially polarized radiation on the basis of information theory. $\backslash \backslash$ Optical methods for studying flows: XV Int. science and technology. conference [Electronic resource]: proceedings of the conference. - Electron. dat. - M.: national research UNIVERSITY "MPEI", 2019. P. 283-. ISBN.

7. Rinkevichius B. S., Laser Doppler anemometry. - M.: KnoRus. 2017.

8. A. S. Akentyev, A. L. Sokolov, M. A. Sadovnikov, G. V. Simonov. Polarization analysis of the guidance system of quantum optical systems // Optics and spectroscopy Vol. 122, № 6, Moscow, 2017 p. 1044-1050

9. A.L. Sokolov. Optical vortices with axisymmetric polarization structure," Optical Engineering № 56(1), 31 January, 2017.

10. Ahmetov I. I., Frolov P. N., Rinkevichius B. S., Kiselev A. V., Tavrov A. V. Achromatic interferometer for $3 \mathrm{D}$ monitoring and analysis of quality of wave front // Meas.Tech.: No. 8. 2013. P.28-30.

11. Frolov P. N., Anan'eva, V. I., and Ksanfomaliti, L. V., Tavrov A. V. Observation of phase curves of exoplanets using stellar coronagraph. Astronomical Bulletin. Vol. 49. No. 6. 2015. P. 448. 